\title{
Resource-Saving Method of Forming Information Infrastructure of Sorting Stations
}

\author{
Anatolii Kosolapov ${ }^{*}$ \\ Dnipro National University of Railway Transport named after academician V. Lazaryan, Computer Department, Ukraine
}

\begin{abstract}
The paper proposes a resource-saving method of conceptual design of the information infrastructure of railway objects on the example of a distributed computer control system of a marshalling yard. This method allows determining the economically optimal degree of decentralization of the technical structure of the management system.
\end{abstract}

\section{Introduction}

The current stage of development of industrial and transport systems is characterized by a grace of technologies and automated functions based on the territorial distribution of resources and the wide use of artificial intelligence methods for solving problems of energy and technical resources, logistics and environmental safety. The integration of processes and systems leads to a synergistic effect and the formation of large intelligent transport systems [1]. In such systems, an important parameter affecting their efficiency is the multi-structure and its component - the technical structure [2]. For railway objects, information infrastructure, including servers, computers, controllers and information channels, are of particular importance. This is due to economic and environmental problems arising in case of failure of individual elements of the structure. For example, for a hump yard it could be a wagon battle, damage to environmentally hazardous goods, and so on. These circumstances increase the role of conceptual design of the information infrastructure of railway objects and use in the development process of resource-saving methods of system design [2].

The paper proposes such a method to determine the rational degree of decomposition of the computer system of the marshalling yard, minimizing production losses due to the unreliability of equipment.

The development of automation systems for marshalling yards in Ukraine and abroad was based on the creation of the first centralized computer systems based on mini-computers (SM-2) to build functionally distributed systems based on industrial microcomputers and microcontrollers (micro-DAT, SM-1800/1810, Advantech microcontrollers) [3, 4]. With the transition to decentralized (hierarchical) systems with a variety of options for distributed structures, the issue of the justification for the transition to decentralized management remains unresolved. Despite numerous attempts to find an answer to this question $[5,6,7,8]$, it remains relevant in the development of technical means of automation of hump yard. When considering the optimization of the structure of the hierarchical system, we will assume that the hump yard, as a technological object of control of the marshalling yard, has local automation devices. The centralized control system, optimizing the overall work of the hump yard, adjusts the work of local automation devices, forming adjustments for regulators (for example, retarder positions and switch operating apparatus). On this principle, the ASC was built for the Yasinovata station of the Donetsk railway [3].

With the growth of power of management computers (MC) there is an interest to consider the expediency of transferring the functions of local automation devices to higher-rank microcontrollers that provide local optimization of the subsystems [8].

The possibilities of automation systems were limited in terms of the implementation of complex control algorithms earlier, and MC did not differ greatly, then there are now technical possibilities to increase the power of both microcontrollers and MC. Therefore, the transfer of functions of local regulators on $\mathrm{MC}$ does not significantly affect its power (memory, speed, etc.).

However, such centralization of management significantly increases the responsibility of MC. Obviously, the failure of the $\mathrm{MC}$ violates the management of a whole hump yard, which leads almost always to great economic losses. Therefore, many authors consider $[9,10,11]$, which determines the reliability of $\mathrm{MC}$ in the solution of this issue.

\section{Problem statement and method of its solution}

The hump yard consists of $\mathrm{n}$ controlled objects $M_{i}$, whose status $\mathrm{O}_{i}$ can be characterized by two parameters: $y_{i}$ - adjustable parameter; $x_{i}$ - control 
action $\left(z_{j}\right.$ - controlled, $f_{g}$ - uncontrolled parameters, see Fig. 1).

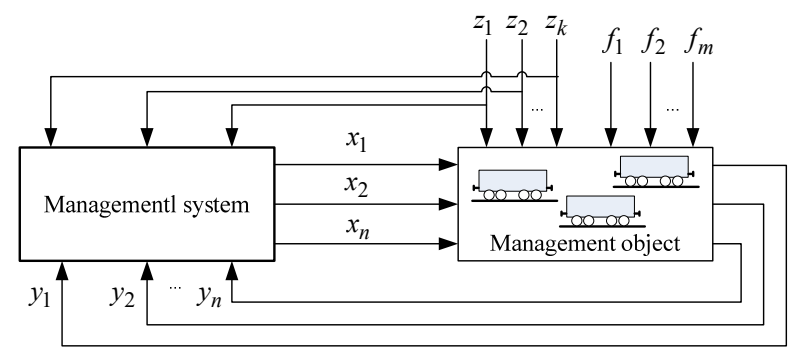

Fig. 1. A marshalling yard as a control object

Thus, the state of the system at each instant of time can be characterized by vectors: the state of the regulated parameter $Y=\left\{y_{1}, \ldots, y_{n}\right\}$; of the position of the regulating body $X=\left\{x_{1}, \ldots, x_{n}\right\}$ and the vector-setpoint $Y^{\prime}=\left\{y_{1}^{\prime}, \ldots, y_{n}^{\prime}\right\}$. The vector-set $Y^{\prime}$ is a task in support of a regulated parameter and in general can differ from its true value - the vector $Y$. If the system does not have local regulators, then $\mathrm{MC}$ directly forms the vector $\mathrm{X}$ and issues it to the executive bodies. In this case, we have a centralized system (Fig. 2).

In the case of local regulators (LCs) implementing local control functions, MC's functions are simplified to formulate only the settings $Y^{\prime}$ for the LC. In this case, the hierarchical structure of the MS is discussed (Fig. 3).

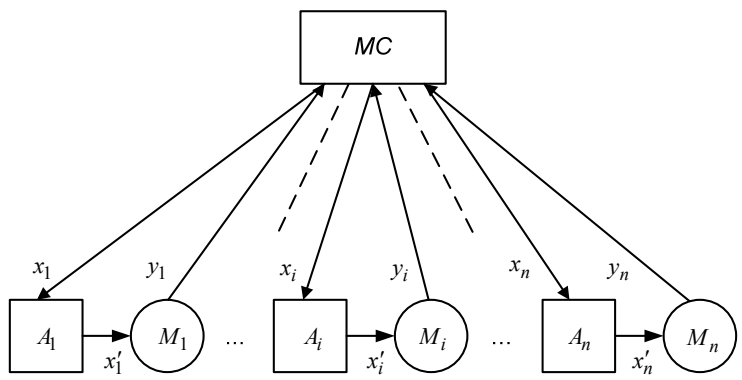

Fig. 2. The structure of the centralized control system

Let the system consist of MC and $n$ local subsystems. In Fig. 2 and Fig. 3 the following symbols are acceptable: Managing System (MS), Actuator (A), Local Controller (LC), Executive Mechanism (M).

The implementation of the management algorithm is reduced to minimizing (maximizing) the function of the species

$$
B=\varphi(Y, X)
$$

(for example, minimizing the deviation of the speed of exit from the retarder position from the set by the control algorithm).

Optimization involves searching for such an optimal value of the control vector $\mathrm{U}^{*}$, so that the management function (1) at the given X takes the optimal value

$$
B^{*}=\min (\max ) \varphi(Y, X)
$$

or

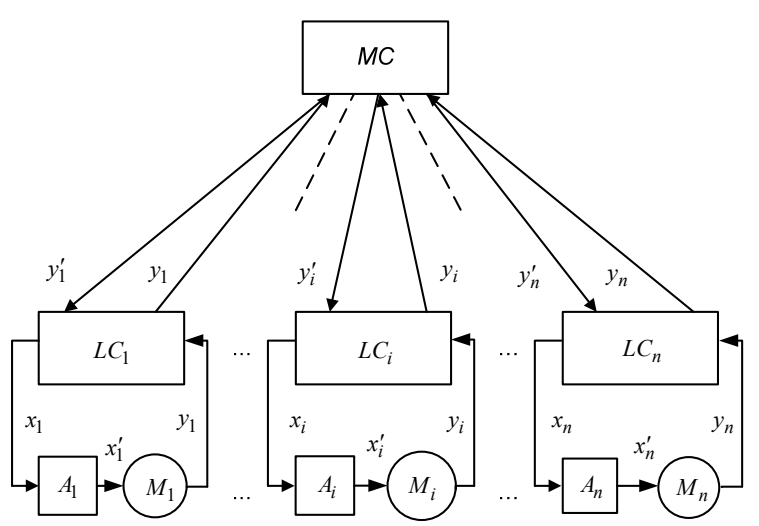

Fig. 3. Structure of the hierarchical system with local control subsystems

The evaluation of the variants of structures will be carried out on the criterion of the full cost of resources. To simplify the calculations we assume that all $\mathrm{n}$ control circuits are the same, the costs of operating systems are also the same and the unreliability of executive bodies can be neglected.

System management will be presented in the mode of time distribution as follows. According to the scheme in Fig. 2 (centralized control system) MC receives information about the state of the controlled object in the form of vectors $\mathrm{Y}$ in cycles in time $T_{c}\left(T_{c}, 2 T_{c}, \ldots, k T_{c}\right)$ intervals. As a result of information processing by algorithm (2), MC carries out the calculation of the new setpoint $Y^{\prime}$ and issuance of the control command to the executive bodies - a vector of the $\mathrm{X}$ position.

According to the scheme in Fig. 3 (hierarchical control system), which also receives information in the form of vectors Y. However, in this case, MC issues only a vector setting $Y^{\prime}$. The calculation of the vector $\mathrm{X}$ is carried out by the local regulators LC; MCM only coordinates their work by adjusting the vector setting $Y^{\prime}$.

Let's see what happens in the case of equipment failure in the kth time interval. In case of failure of MC in a centralized structure, the following situations are possible:

1) the work of the car classification is immediately terminated. Losses $p_{0}$ are determined by the downtime of the object and can be estimated as a complete loss of efficiency during the elimination of the malfunction (system recovery) $T_{\text {rec }}$ :

$$
p_{0}=B^{*} T_{\text {rec }}
$$

2) For some time after the failure of $\mathrm{MC}$ the system continues to function normally and then there is a violation of its work. In this case, the behaviour of the object may vary depending on the position in which the executive bodies will be. They can:

a) Remain in the position in which they were at the moment of the accident $t_{0}$, ie $X=X_{0}$; 
b) Translated into a state of emergency $X=X_{\text {em }}$ characterized by values.

In the second situation, the damage $p_{1}$ will consist of two parts (Fig. 4), $y_{i}=v_{i}$ - speed of exit from the i-th braking position, $\left.v_{i \text { min }}<v_{i}(t)<v_{i \max }\right)$.

At the moment time $t_{1}$, it is proportional to the difference between the optimal and actual values of efficiency:

$$
p_{11}=\left(B^{*}-B_{1}\right) t_{1} .
$$

At the moment time $t_{2}$ there is an output parameter $v_{i}(t)$ at the permissible limits $v_{i}(t)>v_{i \max }$ and local protection disables the object, i.e., losses in this area $p_{12}$ are determined by the total loss of efficiency:

$$
p_{12}=B^{*} t_{2} \text {. }
$$

Complete losses for the second situation

$$
p_{1}=p_{11}+p_{12}=\left(B^{*}-B_{1}\right) t_{1}+B^{*} t_{2},
$$

where

$$
T_{\text {rec }}=t_{1}+t_{2} ; \lim _{t_{1} \rightarrow \infty} p_{1}=B^{*} T_{r e c}
$$

$B_{1}$ - Efficiency in case of not optimal control in the centralized system (manual control of sorting out of cars).

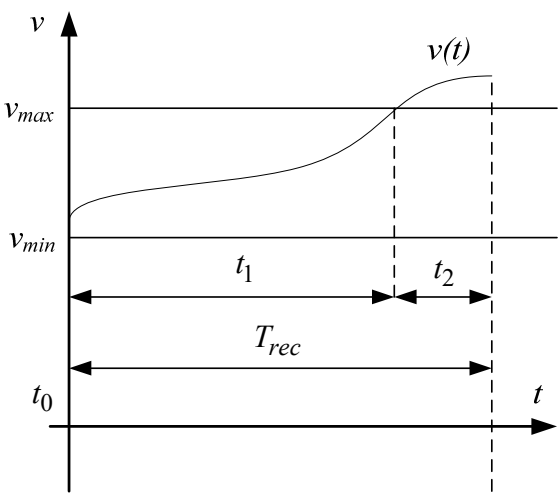

Fig. 4. Example of the behavior of the regulated parameter $v(t)$ within (vin, vmax)

In the hierarchical system, when MC refuses, the control is not lost completely, only its effectiveness is reduced. As a result of the refusal, she takes on meaning $B_{2}$. In this case, the loss from the loss of optimal control

$$
p_{2}=\left(B_{2}^{*}-B_{2}\right) T_{r e c} \text {. }
$$

This damage, as a rule, is much less than the damage that is obtained when a CM system fails in a centralized system. Reducing the damage to a system with a hierarchical structure is achieved by increasing its value due to the presence of local regulators. In addition, the effect of loss reduction is reduced due to additional losses caused by the unreliability of local regulators. Let's take the following for further analysis

Assumption:

$B^{*}$ - Loss per unit time when the car classification does not work;

$B_{1}$ - Loss per unit of time when the centralized system does not work MC (non-optimal control);

$B_{2}$ - Loss per unit of time when the hierarchical system does not work MC (non-optimal control);

$b_{i}^{*}$ - Loss per unit of time when the i-th control circuit is not working

$b_{i}$ - Loss per unit time with a sub-optimal value of the efficiency of the i-th circuit.

$$
B^{*}>B_{1}>B_{2} \gg b_{i}^{*}>b_{i} .
$$

Then the full costs for centralized $\mathrm{P}_{1}$ and hierarchical $\mathrm{P}_{2}$ systems can be estimated as follows:

$$
\mathrm{P}_{1}=\left(B^{*}-B_{1}\right) \lambda_{0} t_{1}+B^{*} \lambda_{0} t_{2}+C_{0}^{\mathrm{centr}}
$$

$$
\begin{aligned}
& \mathrm{P}_{2}=\left(B^{*}-B_{2}\right) \lambda_{0} T_{r e c}+ \\
& +\sum_{i=1}^{n}\left[\left(b_{i}^{*}-b_{i}\right) \lambda_{i} t_{i 1}+b_{i}^{*} \lambda_{i} t_{i 2}\right]+C_{0}^{d e c}+\sum_{i=1}^{n} c_{i},
\end{aligned}
$$

where $\lambda_{0}$ - the intensity of bugs CM in hierarchical and centralized systems; $\lambda_{i}$ - intensity of failure of the i-th local regulator; $C_{0}^{c e n}, C_{0}^{d c e n}, c_{i}$ - the cost of $\mathrm{CM}$ respectively in the centralized system, in a decentralized (hierarchical) system and the cost of the microcontroller system of the i-th local regulator.

We will assume that

$$
C_{0}^{c e n}>C_{0}^{d c e n} \gg c_{i} .
$$

To simplify further analysis, we assume that the system is homogeneous in its composition, i.e.

$$
b_{i}=b_{n} ; \lambda_{i}=\lambda_{n} ; c_{i}=c_{n} ; i=1,2, \ldots, n \text {. }
$$

For the i-th control circuit, taking into account the possible failure of the regulator by analogy with the centralized control system (Fig. 4), we can write

$$
t_{i 2}=T_{r e c i}-t_{i 1}
$$

where $t_{i 2}$ - the time during which the i-th parameter is within the permissible values; $T_{\text {reci }}$ - time of repair of the i-th regulator; $t_{i 1}$ - the time during which the i-th parameter does not exceed the permissible limits.

In the first approximation we will accept that

$$
\begin{aligned}
& t_{i 1}=t_{n 1}=t_{1}, i=1,2, \ldots, n ; \\
& T_{r e c}^{c e n}(\mathrm{CM})=T_{r e c}^{d e c}(\mathrm{CM})=T_{r e c}\left(c_{n}\right) .
\end{aligned}
$$


For further research, we introduce the coefficient

$$
t_{1}=r T_{r e c}, t_{2}=(1-r) T_{r e c} .
$$

Then we have from (6) and (7)

$$
\begin{aligned}
& \mathrm{P}_{1}=\left(B^{*}-B_{1}\right) \lambda_{0} r T_{r e c}+B^{*} \lambda_{0}(1-r) T_{r e c}+C_{0}^{c e n}, \\
& \mathrm{P}_{2}=\left(B^{*}-B_{2}\right) \lambda_{0} T_{r e c}+\left(B^{*}-B_{1}\right) \lambda_{n} r T_{r e c}+ \\
& +B^{*} \lambda_{n}(1-r) T_{r e c}+C_{0}^{d e c}+n c_{n} .
\end{aligned}
$$

Let's turn to the integral index of reliability - the readiness factor

$$
\begin{gathered}
K_{\text {avail }}^{\text {cen }}(\mathrm{CM})=K_{\text {avail }}^{\text {dcen }}(\mathrm{CM})=K_{0}= \\
=\frac{T_{m t f}}{T_{m t f}+T_{\text {rec }}}=\frac{1 / \lambda_{0}}{1 / \lambda_{0}+T_{\text {rec }}}, \\
K_{\text {avail }}\left(c_{n}\right)=K_{n}=\frac{T_{\operatorname{mtt}(n)}}{T_{\operatorname{mtf}(n)}+T_{\text {rec }}}=\frac{1 / \lambda_{n}}{1 / \lambda_{n}+T_{\text {rec }}},
\end{gathered}
$$

where $T_{m t f}$ - mean time to failure and

$$
\lambda_{0}=\frac{1-K_{0}}{K_{0} T_{\text {rec }}} ; \lambda_{n}=\frac{1-K_{n}}{K_{n} T_{\text {rec }}} .
$$

Then let's introduce the coefficient $\alpha$ :

$$
K_{n}=\alpha K_{0} .
$$

We substitute (14) and (15) in (10), (11), normalize full costs and introduce the notation

$$
\delta_{1}=\frac{B^{*}-B_{1}}{B^{*}} ; \delta_{2}=\frac{B^{*}-B_{2}}{B^{*}} .
$$

As a result, we get

$$
\begin{aligned}
& \tilde{\mathrm{P}}_{1}=\frac{\mathrm{P}_{1}}{B^{*}}=\frac{\left(1-K_{0}\right)\left(1+r\left(\delta_{1}-1\right)\right)}{K_{0}}+\frac{C_{0}^{c e n}}{B^{*}}, \\
& \tilde{\mathrm{P}}_{2}=\frac{\mathrm{P}_{2}}{B^{*}}=\frac{\delta_{2}\left(1-K_{0}\right)}{K_{0}}+ \\
& +\frac{\left(1-\alpha K_{0}\right)\left(1+r\left(\delta_{1}-1\right)\right)}{\alpha K_{0}}+\frac{C_{0}^{d c e n}+n c_{n}}{B^{*}},
\end{aligned}
$$

In Fig. 5, constructed graphs $\tilde{\mathrm{P}}_{1}=f_{1}\left(K_{0}\right)$ and $\tilde{\mathrm{P}}_{2}=f_{2}\left(K_{0}\right)$ under the following assumptions $r=0,9$ and $\alpha=1,1$.

From the graphs it follows that with the increase of the coefficient of readiness of loss in the hierarchical decentralized system, in comparison with the centralized decreases $\left(K_{0}^{(1)}, K_{0}^{(2)}, K_{0}^{(3)}\right.$ - points on the graph where losses in both systems are the same). From (17) it follows that the losses in the hierarchical system are linearly dependent on the number and cost of local microcontroller regulators. Given the reliability indices
$\left(K_{0}, \alpha\right)$ and the value of the subsystems in the technical structure of the control system $\left(C_{0}^{c e n}, C_{0}^{d c e n}\right.$ and $\left.c_{n}\right)$, the number of contours of local control $\mathrm{n}$ can be chosen based on the solution of equations (16) and (17).

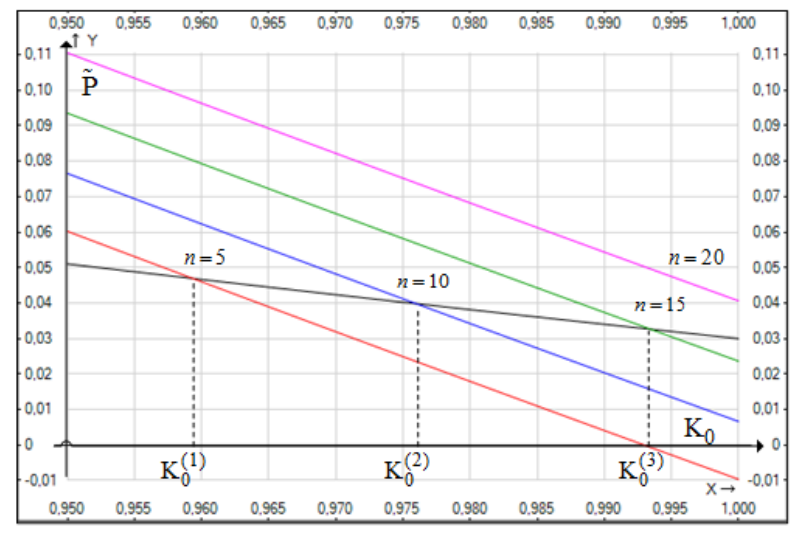

Fig. 5. Dependence of change of systems efficiency on reliability of $\mathrm{MC}$ and $\mathrm{LC}$

If we compare (16) and (17), then introduce normalized coefficients

$$
a_{1}=10^{-3} \delta_{2} B^{*} ; a_{2}=10^{-3} c_{n} ; a_{3}=10^{-3}\left(C_{0}^{d e c}-C_{0}^{c e n}\right),(18
$$

we can obtain the necessary equations of two variables $K_{0}$ and n:

$$
a_{1}\left(1-K_{0}\right) / K_{0}+a_{2} n+a_{3}=0 .
$$

Fig. 6, 7, 8 shows the planes that represent the geometric point of the points $K_{0}^{(i)}$ in which the losses in the centralized and hierarchical (decentralized) systems are identical and corresponds to the change of priorities in the construction of systems, depending on the readiness coefficients and the number of local regulators. Analysis of the results shows that rational solutions are located in the zone of high readiness and a small number of local subsystems. The zone of rational decisions increases if the value of the coefficient $a_{1}$ decreases. The number of local circuits does not exceed 15 subsystems.

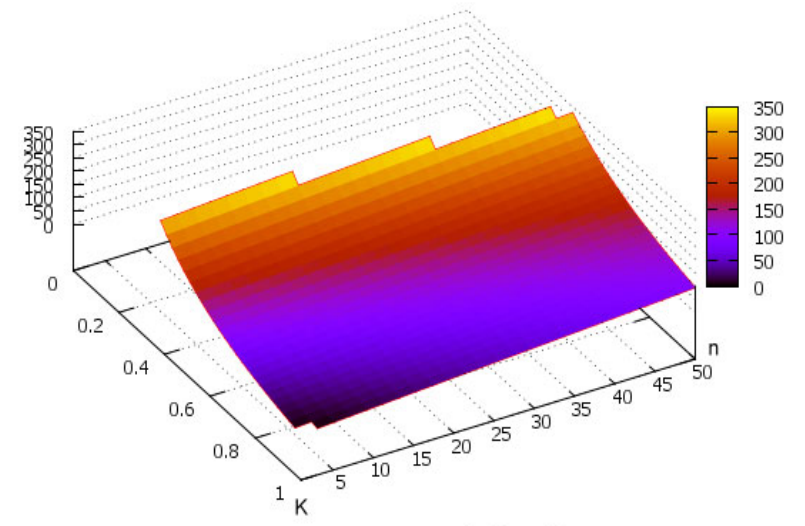

Fig. 6. Plans of equal losses of centralized and hierarchical systems $\left(a_{1}=200\right)$ 


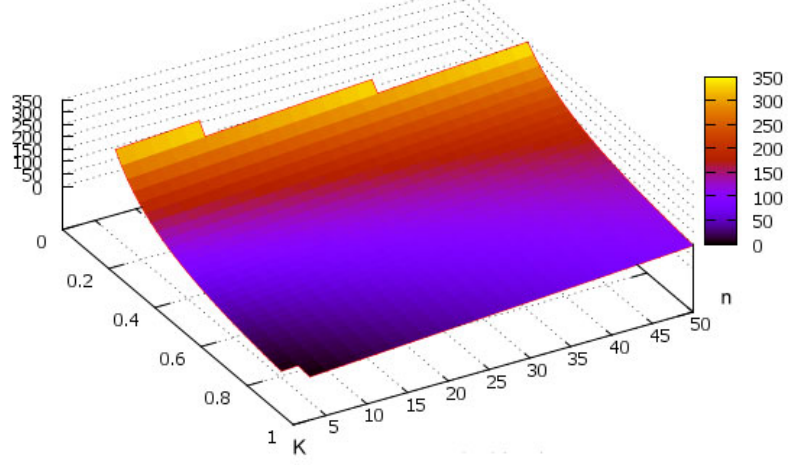

Fig. 7. Plans for $a_{1}=100$

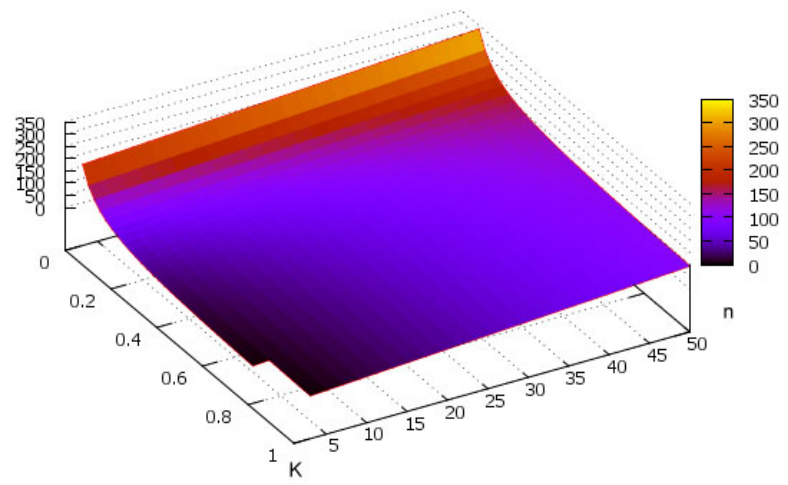

Fig. 8. Plans for $a_{1}=10$

\section{Conclusions}

It should be borne in mind that the results obtained are valid only for the case of finite and additive losses. For objects, the complete refusal of automatic devices which can lead to accidents such as catastrophes or associated with the possibility of human victims, the choice undoubtedly must be resolved in favor of the combined system.

\section{References}

1. Kosolapov A., Loboda D. Semiotic-AgentOntological Approach To Design Intellectual Transport Systems. American Journal Of
Engineering Research (Ajer), Volume-7, Issue-7, pp.205-209 (2018)

2. Kosolapov A,, Loboda D. Framework Conceptual Design Of Complex Real-Time Management System (Codecs). European Journal of Advances in Engineering and Technology, 5(8), pp. 559-566 (2018)

3. A. A. Kosolapov, A. L. Blokhin, K. F. Boryak, et al. The key role of transport in the modern world: a monograph - Odessa: KUPRIENKO SV - 163 p. (2013)

4. Automation Device and Computing 2012-2013 [Electronic resource] / Access mode: http://support.advantech.com.tw/ OnlineResources / SubIndex.aspx? $\mathrm{Bu}=$ 99B2E2BE-A7E3-4C589E35-B4F48A952AC7 \& type $=$ e Catalog. : Advantech (2013)

5. G.A. Krasovsky, L.V. Safris, A.A. Kosolapov, B.M. Filimonov, E.M. Shafit, E.V. Shcherbakov. Patent № 1073146, MKI, B61, L17 / 00. A device for controlling the technological process of the dissolution of compositions on a sorting chamber. Bul. №6. - USSR (1984)

6. V.I. Ivanchenko, N.N. Lyabakh, A.A. Sepety $A$ new approach to managing the process of dissolution of trains on a hump yard. Works of RIIZhT. - Rostovon-Don. pp. 34-41 (1984)

7. E. M. Shafit, I. V. Zhukovitsky, A. A. Kosolapov. Development of a unified system of automated control of the process of disbanding trains at a sorting station. Materials of temporary teams. St. Petersburg. pp. 81-93 (1996)

8. G. A. Shastova, A. I. Koykin. Selection and optimization of the structure of information systems. M.: Energy, 256 p. (1972)

9. Nechiporenko V.I. Structural analysis of systems (efficiency and reliability). M.: Soviet radio. 216 p. (1977)

10. A. M. Polovko, S. V. Gurov. Fundamentals of the theory of reliability. $\mathrm{SPb}$. BHV-Petersburg.704 p. (2008)

11. Fritch V. Application of microprocessors in control systems: Trans. with him. M.: Mir,.464 p. (1984) 